\title{
Adrenergic regulation of monocyte chemotactic protein 1 leads to enhanced macrophage recruitment and ovarian carcinoma growth
}

\author{
Guillermo N. Armaiz-Pena ${ }^{1}$, Vianey Gonzalez-Villasana ${ }^{2}$, Archana S. Nagaraja ${ }^{1}$, \\ Cristian Rodriguez-Aguayo ${ }^{2}$, Nouara C. Sadaoui ${ }^{1}$, Rebecca L. Stone ${ }^{3}$, Koji Matsuo ${ }^{4}$, \\ Heather J. Dalton ${ }^{1}$, Rebecca A. Previs ${ }^{1}$, Nicholas B. Jennings ${ }^{1}$, Piotr Dorniak ${ }^{1}$, \\ Jean M. Hansen ${ }^{1}$, Jesusa M.G. Arevalo ${ }^{5}$, Steve W. Cole ${ }^{5}$, Susan K. Lutgendorf ${ }^{6}$, \\ Anil K. Sood ${ }^{1,7,8}$, Gabriel Lopez-Berestein ${ }^{2,7,8}$

\footnotetext{
${ }^{1}$ Department of Gynecologic Oncology and Reproductive Medicine, The University of Texas M. D. Anderson Cancer Center, Houston, Texas 77030, USA

${ }^{2}$ Department of Experimental Therapeutics, The University of Texas M. D. Anderson Cancer Center, Houston, Texas 77030 , USA

${ }^{3}$ Department of Obstetrics and Gynecology, The John Hopkins University, Baltimore, MD 21287, USA

${ }^{4}$ Division of Gynecologic Oncology, Department of Obstetrics and Gynecology, University of Southern California, Los Angeles, CA 90089, USA

${ }^{5}$ Department of Medicine, Division of Hematology-Oncology, University of California, Los Angeles, CA 90095, USA

${ }^{6}$ Department of Psychology, Obstetrics and Urology, and Holden Comprehensive Cancer Center, University of Iowa, Iowa City, Iowa 52242, USA

${ }^{7}$ Department of Cancer Biology, The University of Texas M. D. Anderson Cancer Center, Houston, Texas 77030, USA

${ }^{8}$ Center for RNA Interference and Non-coding RNA, The University of Texas M. D. Anderson Cancer Center, Houston, Texas 77030, USA
}

Correspondence to:

Gabriel Lopez-Berestein, e-mail: glopez@mdanderson.org

Keywords: ovarian cancer, macrophages, monocytes, catecholamines, MCP1

Received: September 15,2014 Accepted: December 09, $2014 \quad$ Published: December 27, 2014

\section{ABSTRACT}

Increased adrenergic signaling facilitates tumor progression, but the underlying mechanisms remain poorly understood. We examined factors responsible for stress-mediated effects on monocyte/macrophage recruitment into the tumor microenvironment, and the resultant effects on tumor growth. In vitro, MCP1 was significantly increased after catecholamine exposure, which was mediated by CAMP and PKA. Tumor samples from mice subjected to daily restraint stress had elevated MCP1 gene and protein levels, increased CD14+ cells, and increased infiltration of CD68+ cells. hMCP1 siRNA-DOPC nanoparticles significantly abrogated daily restraint stressinduced tumor growth and inhibited infiltration of CD68+ and F4/80+ cells. In ovarian cancer patients, elevated peripheral blood monocytes and tumoral macrophages were associated with worse overall survival. Collectively, we demonstrate that increased adrenergic signaling is associated with macrophage infiltration and mediated by tumor cell-derived MCP1 production.

\section{INTRODUCTION}

Sustained adrenergic activation resulting from prolonged behavioral stress leads to increased tumor growth $[1,2]$. Chronic increases in pro-tumoral catecholamines, norepinephrine and epinephrine, affect a broad array of processes involved in the progression and metastasis of various cancers [3]. Sympathetic nervous system activity directly influences the pathogenesis of ovarian carcinoma by protecting tumor cells from anoikis [4], promoting tumor cell invasion [5-7] and increasing tumor-associated angiogenesis $[1,8]$. These data highlight the importance of the tumor microenvironment in these processes, but the underlying mechanisms require further analysis.

Tumor associated macrophages (TAMs) are key components of the tumor microenvironment [9] and reflect 
a continuum of maturation/differentiation of bone marrow derived monocyte precursors that adapt to the different microenvironments where they will eventually reside [10]. They differentiate from circulating blood monocytes recruited into the tumor microenvironment and are rapidly activated by tumor cells. Activated TAMs release cytokines, chemokines, enzymes, and pro-inflammatory factors that lead to further macrophage recruitment and increased tumor growth [11]. Recently, it has been reported that patients with higher levels of adrenergic signaling have higher counts of MMP-9-producing TAMs [12]. Additionally, in an orthotopic mouse model of breast cancer, restraint stress was shown to induce breast cancer metastasis, largely mediated by increased macrophage infiltration into the tumor [13]. While several studies have demonstrated that increased adrenergic signaling leads to enhanced macrophage recruitment into the tumor, the underlying mechanisms remain poorly understood. We describe an ADRB/PKA axis that mediates increased MCP1 levels resulting in enhanced macrophage infiltration and tumoral growth. Furthermore, we demonstrate that increased peripheral blood monocytes and TAMs are associated with advanced disease and decreased overall survival in ovarian cancer patients.

\section{RESULTS}

\section{NE and Epi increase ovarian cancer cell derived- MCP1 production}

To elucidate the mechanism used by tumor cells to induce monocyte/macrophage recruitment while undergoing sustained adrenergic signaling, we analyzed chemokine/cytokine changes using a cytokine/chemokine panel generated from SKOV3ip1 cells treated with norepinephrine or epinephrine. Several cytokines, such as fractalkine, IL6, IL8, MCP1 and VEGF were identified to be upregulated by both catecholamines (Supplementary Table 1), while other key players in the induction of monocyte/macrophage recruitment, such as CSF1, were not found to be elevated after NE treatment (Supplementary Table 1 and Supplementary Figure 1a). Hence, we decided to focus on MCP1 due of its known role in the induction of macrophage recruitment into the microenvironment.

We examined the expression pattern of MCP1 in an ovarian cancer cell panel. RT-PCR results show that MCP1 expression varied among all cell lines tested, with SKOV3ip1, A2780-CP20, SKOV3ip1-TR and 2774 cells being MCP1 positive (Supplementary Figure 1b). Next, we examined if norepinephrine or epinephrine stimulation could directly increase MCP1 production by ovarian cancer cells. To confirm that SKOV3ip1 cells respond to NE treatment, we determined cAMP levels by ELISA. cAMP levels were significantly increased at 15 and $30 \mathrm{~min}$ after NE treatment (Supplementary Figure 1c). A significant dose-dependent increase in MCP1 gene production was seen in SKOV3ip1 cells at 3 hours following NE treatment (Figure 1a). This gene increase peaked at 3 hours and subsequently returned to baseline (Figure 1b). To ascertain that gene stimulation was followed by protein production, we further analyzed supernatant obtained from SKOV3ip1 cells treated with NE or Epi. MCP1 protein secretion was significantly enhanced after treatment with both catecholamines (Figure 1c). This same effect was seen after treatment with isoproterenol, an ADRB agonist (Figure 1c). To determine which adrenergic receptor mediate NE-induced MCP-1 gene expression, we treated SKOV3ip1 cells with propranolol (non-specific beta-blocker), atenelol (ADRB1), ICI115,881 (ADRB2) or SR59230A (ADRB3) and added NE for $3 \mathrm{hrs}$. A significant increased in MCP1 gene expression was seen after NE exposure and this effect was abrogated by propranolol and ICI115,881 (Figure 1d). Since downstream ADRB signaling can be largely mediated by increased cAMP and PKA activity, we treated SKOV3ip1 cells with forskolin (cAMP agonist) or dbcAMP (PKA agonist). Results demonstrated that forskolin or dbcAMP could induce cancer cell-derived MCP1 production (Figure 1e). As a whole, these data suggest that catecholamines can induce MCP1 production through an ADRB/cAMP/PKA signaling axis.

\section{Restraint stress leads to increased tumor cell- derived MCP1 production, monocyte and macrophage recruitment}

We next investigated if increased adrenergic signaling could enhance tumor derived MCP1 production in vivo. To induce tumoral catecholamine levels, we used a well-characterized restraint stress model $[1,14]$. Mice inoculated with SKOV3ip1 cells were subjected to daily restraint stress and tumor samples were analyzed for MCP1 gene and protein levels. After three weeks of restraint stress, a significant induction of MCP1 gene expression levels was observed (Figure 2a). In this same model, we observed a significant week-by-week increase in MCP1 gene expression, suggesting that MCP1 production is enhanced as the tumor progresses under sustained adrenergic signaling (Supplementary Figure 2a). This increase was significantly associated with elevated tumoral NE (Supplementary Figure 2b-2c). Additionally, tumor samples obtained from mice inoculated with 2774 cells and subjected to daily restraint stress exhibited a significant increase in MCP1 protein (Figure 2b). To determine if increased MCP1 production correlated with monocyte recruitment, we assessed samples for MCP1 and CD14 expression by immunofluorescence. This analysis revealed that restraint stress increased $\mathrm{MCP} 1$ production, and in areas with elevated MCP1 levels, $\mathrm{CD} 14+$ cells were observed (Figure 2c). These samples were also analyzed for the presence of CD68+ cells. We observed an increase in CD68+ cells in samples from mice subjected to daily restraint stress (Figure 2d). 


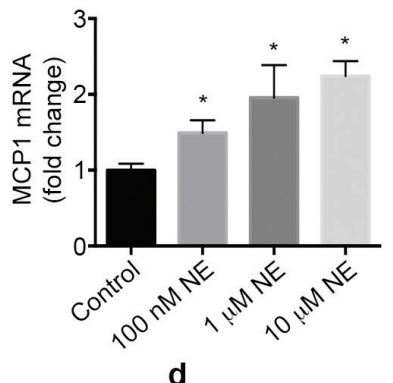

b

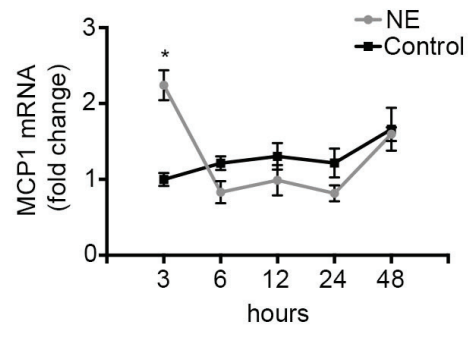

e
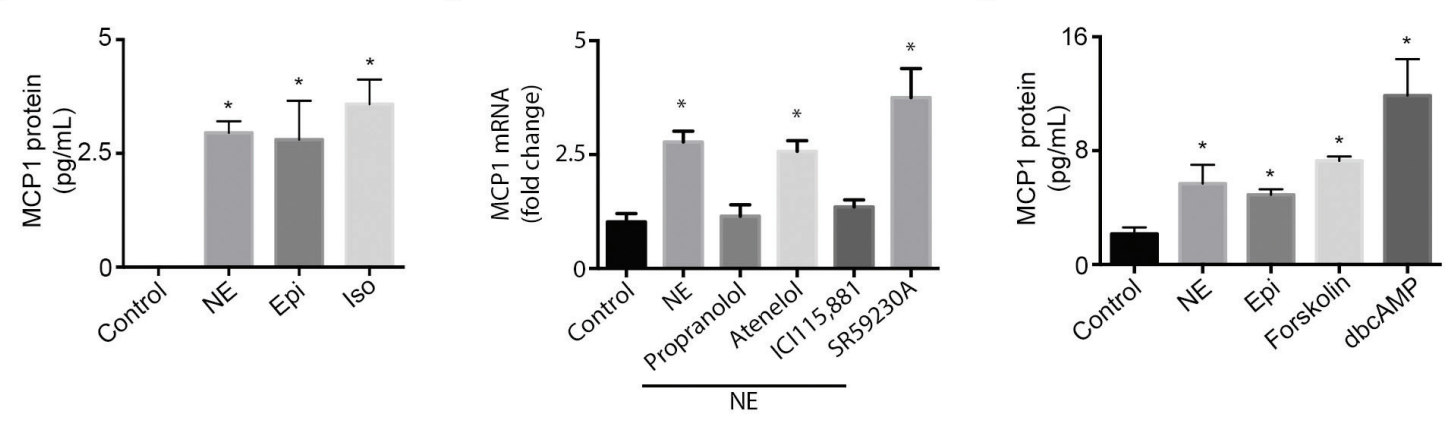

Figure 1: Catecholamines induce MCP1 expression. (a) SKOV3ip1 cell MCP1 gene expression after treatment with several doses of NE for 3 hours. (c) SKOV3ip1 cell MCP1 gene expression after treatment with $10 \mu \mathrm{M}$ NE for various time intervals. (b) MCP1 concentration in medium from SKOV31ip1 cells after treatment with $10 \mu \mathrm{M}$ norepinephrine, $10 \mu \mathrm{M}$ epinephrine and $10 \mu \mathrm{M}$ isoproterenol for $3 \mathrm{hrs}$. (d) MCP1 gene expression after treatment with $10 \mu \mathrm{M}$ propranolol, $10 \mu \mathrm{M}$ atenelol, $1 \mu \mathrm{M}$ ICI115,881 or 10 $\mu \mathrm{M}$ SR59230A followed by $10 \mu \mathrm{M}$ norepinephrine for $3 \mathrm{hrs}$. (e) MCP1 concentration in medium from SKOV3ip1 cells after treatment with $10 \mu \mathrm{M}$ norepinephrine, $10 \mu \mathrm{M}$ epinephrine, $1 \mu \mathrm{M}$ forskolin and $10 \mu \mathrm{M}$ dbcAMP for $3 \mathrm{hrs} . * P<0.05$.

a

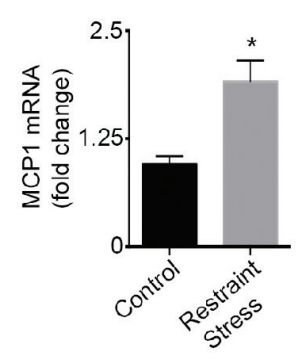

b

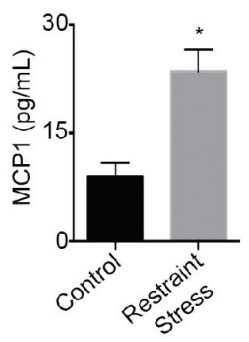

C

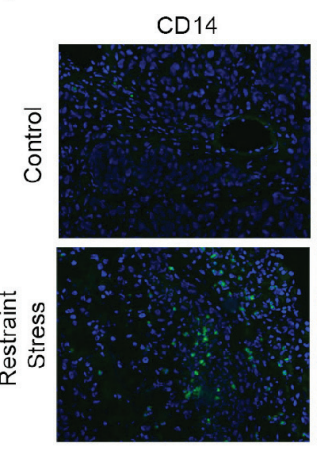

d
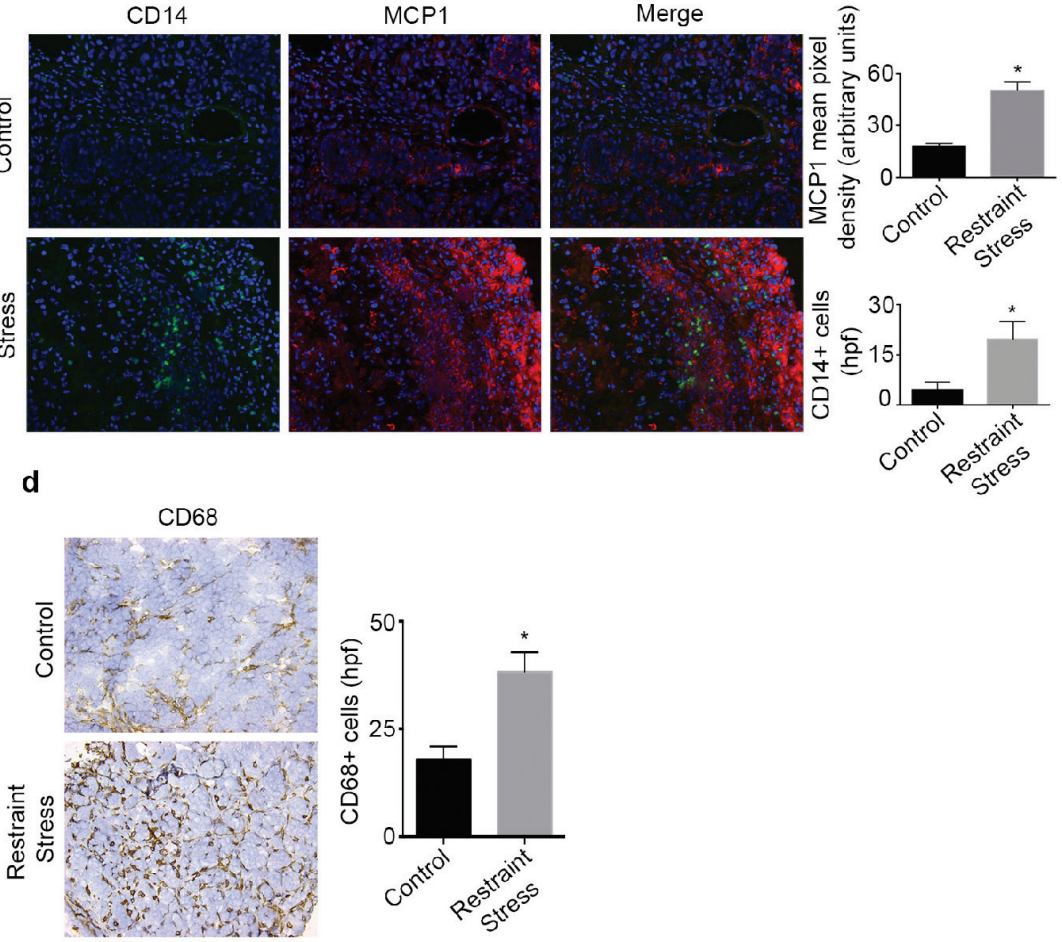

Figure 2: Daily restraint stress leads to increased MCP1 expression and infiltration of CD14+ and CD68+ cells. (a) MCP1 mRNA or (b) protein levels in tumor samples from mice subjected to daily restraint stress. (c) Immunofluorescence analysis of tumor samples from mice subjected to daily restraint stress showing the presence of MCP1 and CD14+ cells. (d) Immunohistochemical analysis of tumor samples from mice subjected to daily restraint stress showing the presence of CD68+ cells. ${ }^{*} P<0.05$. 


\section{Restraint stress requires tumor-derived MCP1 to promote macrophage infiltration and tumor growth}

We next investigated the role of MCP1 in the promotion of macrophage recruitment and tumor growth in response to sustained adrenergic signaling. To induce tumoral catecholamine levels, we used a wellcharacterized restraint stress model. First, we confirmed the efficacy of siRNA for silencing MCP1 in SKOV3ip1 cells (Supplementary Figure 3a). Subsequently, mice inoculated with SKOV3ip1 cells were subjected to daily restraint stress and treated with either control siRNADOPC or a validated hMCP1 siRNA-DOPC. In this model, restraint stress resulted in increased tumor growth, nodule counts and MCP1 levels, while hMCP1 siRNADOPC completely abrogated this effect (Figure $3 a-3 b$ and Supplementary Figure $3 b$ ). The increase in tumor growth in response to daily restraint stress was also accompanied by increased infiltration of $\mathrm{CD} 68+$ cells, but this effect was blocked by hMCP1 siRNA-DOPC (Figure 3c).
Furthermore, restraint stress increased the amount of F4/80+ cells, while hMCP1 siRNA-DOPC abrogated these effects (Figure 3d). Next, we asked if propranolol could block restraint stress induced macrophage infiltration into the tumor. Results show that restraint stress increased the presence of $\mathrm{F} 4 / 80+$ cells, while propranolol blocked this effect (Figure 3e). These data point towards MCP1 as a key driver of restraint stress-induced macrophage recruitment ultimately leading to increased tumor growth.

\section{Elevated peripheral blood monocyte levels and tumoral macrophages are associated with poor outcome in patients with ovarian cancer}

Next, we examined the levels of peripheral blood monocytes in a cohort of ovarian cancer patients $(n=462)$ and studied associations between clinical and pathological variables (Supplementary Table 2). Of the patients evaluated, $28 \%$ had elevated absolute peripheral blood monocyte counts $\left(>0.7 \times 10^{6} / \mu \mathrm{L}\right)$ and $43 \%$ had a

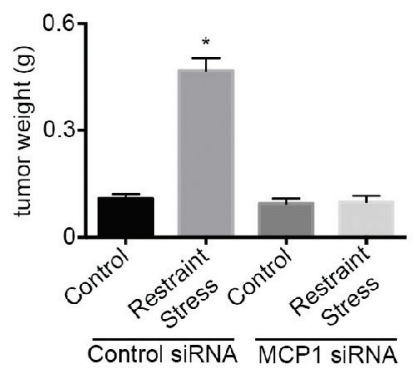

b

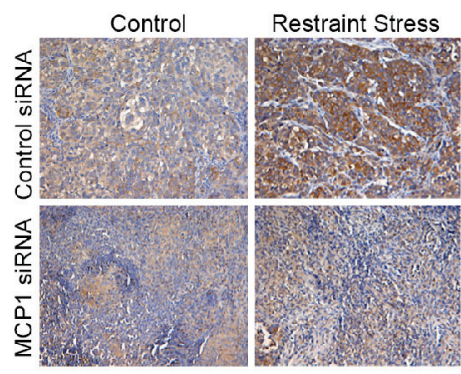

c

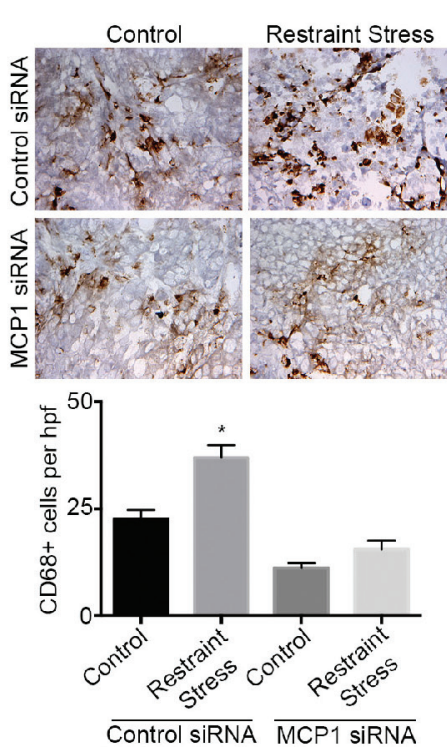

d
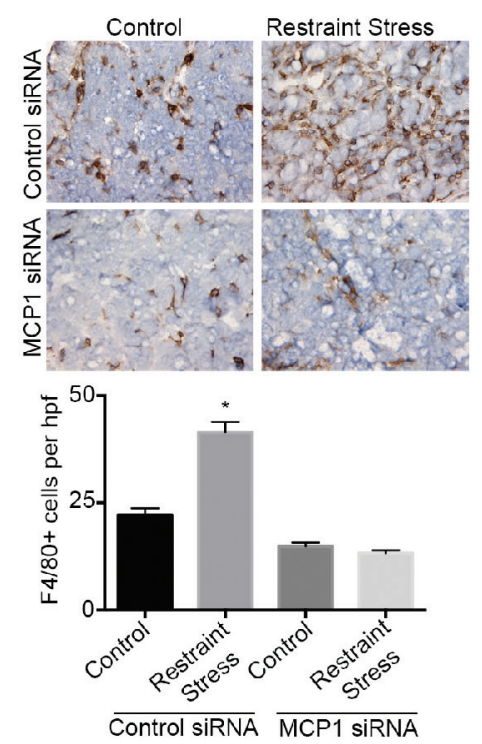

e
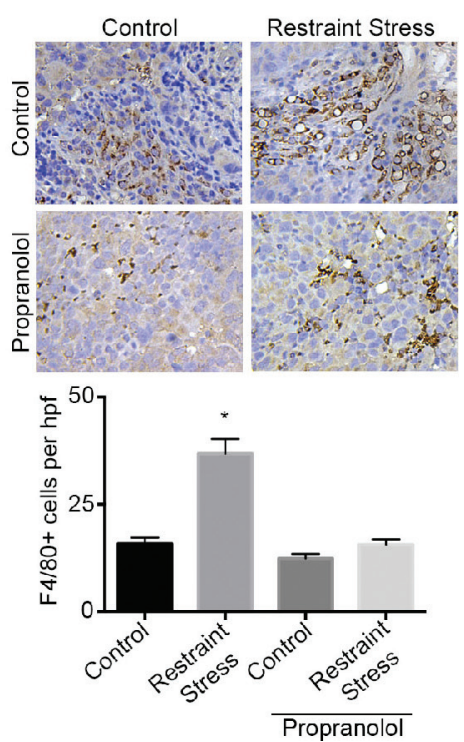

Figure 3: hMCP1 siRNA-DOPC nanoparticles abrogates restraint stress induced increase in tumor growth and macrophage recruitment. (a) Tumor weight in restraint stress orthotopic SKOV3ip1 model treated with control or hMCP1 siRNA-DOPC nanoparticles. (b) MCP1 immunohistochemical analysis of tumor samples. (c) CD68 and (d) F4/80 immunohistochemical analysis of tumor samples. (e) F4/80 immunohistochemical analysis of tumor samples from mice subjected to restraint stress and propranolol. $* P<0.05$. 
high peripheral blood monocytes when measured as the percent of white blood cell content $(>8 \%)$. Elevated peripheral blood monocytes were significantly associated with increased ovarian cancer stage at the time of diagnosis and decreased survival (Figure $4 a-4 b$ and Supplementary Figure $4 a-4 b$ ). A subset of these patients had tumor samples available for assessment of CD68+ cell infiltration $(n=76)$. Of the samples evaluated, 50\% had elevated CD68+ counts (determined as $>15$ per high power field; 20x). High counts of CD68+ cells were significantly associated with decreased overall survival (Figure $4 c-4 d$ ). We carried out a multivariate analysis including CD68+ counts, stage, grade and extent of cytoreduction. In this analysis, elevated CD68+ cell count $(p=0.04)$ and suboptimal cytoreduction $(p=$ 0.003 ) remained significantly associated with poor patient survival. Additionally, peripheral blood monocytes were significantly correlated with increased tumoral CD68+ cells (Supplementary Figure 4c). Furthermore, we looked for correlation between tumoral MCP1 gene levels and elevated CD68+ counts. Our data demonstrates that elevated MCP1 gene levels were correlated with higher CD68+ counts (Supplementary Figure 4d). We also studied the prognostic value of MCP1 mRNA expression using the Kaplan-Meier plotter (http://kmplot.com/analysis/index. php? $\mathrm{p}=$ service \& cancer $=$ ovar), which assesses the effect of 22,277 genes on survival in 1,171 ovarian cancer patients [15]. KM plotter analysis showed that elevated MCP1
mRNA expression had a negative impact on patients' progression free survival (Figure 4e).

\section{DISCUSSION}

We demonstrate that increased adrenergic signaling leads to enhanced macrophage recruitment, which is mediated by tumor cell-derived MCP1. Clinically, elevated peripheral blood monocytes and tumoral macrophages were significantly associated with decreased survival in ovarian cancer patients. To the best of our knowledge, this is the first report to elucidate the underlying mechanism by which ovarian cancer cells, under sustained adrenergic signaling, recruit macrophages into the tumor microenvironment.

Previous work has shown that macrophage infiltration into the tumor microenvironment is chemokine and cytokine driven [9]. One key factor is MCP1, a potent monocyte/ macrophage chemotactic agent [16]. MCP1 is produced by stromal components such as fibroblasts, endothelial cells, monocytes, and as shown in this study, tumor cells [9]. Chronic inflammation, observed in the tumor microenvironment, has been correlated with increased expression of cytokines, including MCP1 [17]. This increase has been associated with enhanced macrophage infiltration and tumor progression in breast cancer [17]. Previous reports have suggested that ovarian cancer cells are a major source of MCP1 and a key player in the recruitment of macrophages [18]. Recently, microarray analyses of tumors

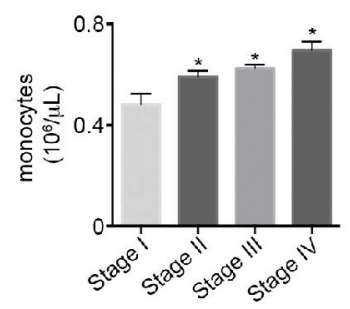

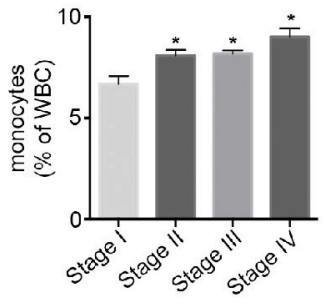

b
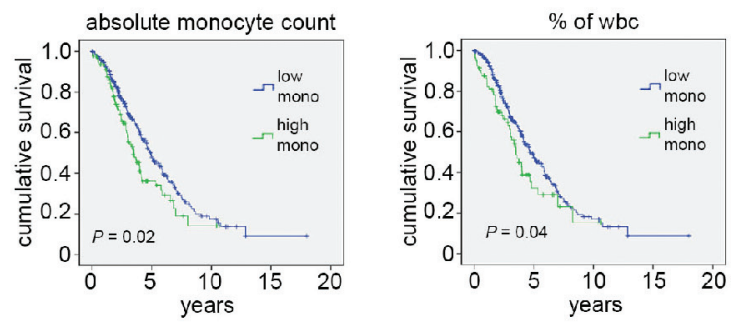

C

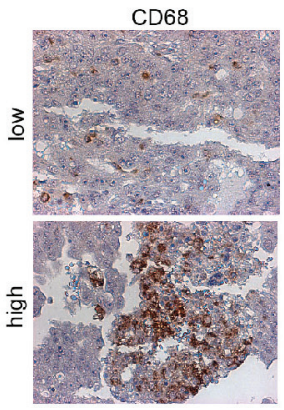

d

e
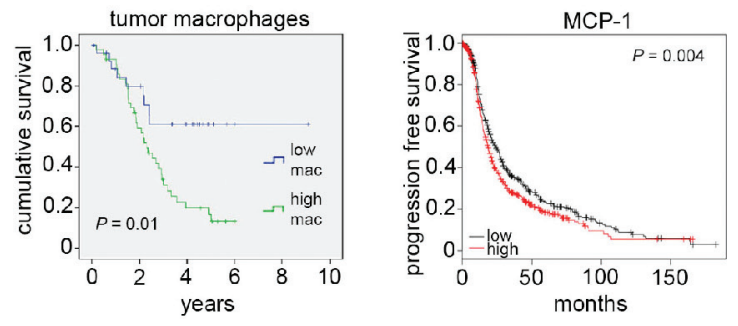

Figure 4: Elevated peripheral blood monocytes and tumoral macrophages correlate with poor outcome in ovarian cancer patients. (a) Peripheral blood monocyte levels by ovarian cancer stage. (b) Kaplan-Meier curves of disease-specific survival in patients with epithelial ovarian carcinoma $(n=462)$ with high or low peripheral blood monocyte levels. (c) Representative picture of CD68 immunohistochemical analysis of patient tumor samples. (d) Kaplan-Meier curve of disease-specific survival in patients with epithelial ovarian carcinoma $(n=76)$ with high or low macrophage counts. (e) Kaplan-Meier curve of relapse free survival in patients with epithelial ovarian carcinoma $(n=1,171)$ with high or low MCP1 mRNA obtained from KM Plotter. $* P<0.05$. 
from ovarian cancer patients at greater biobehavioral risk (i.e., high depression and low social support) revealed an upregulation of MCP1 (reported as CCL2) gene expression. This elevated expression may be correlated to sustained tumoral adrenergic signaling. [19]. Another study demonstrated that tumor samples from ovarian cancer patients with greater biobehavioral risk had higher levels of MMP2+ and MMP9+ macrophage populations [12]. This increase in TAMs may be correlated with higher levels of tumoral adrenergic signaling that could facilitate TAM-induced tumor cell metastasis via increased MMPs release. These data suggest a role for MCP1 as a key mediator of adrenergically-induced macrophage infiltration and ovarian cancer growth.

These findings demonstrate another mechanism by which sustained adrenergic signaling induces tumor growth. Under this condition tumoral catecholamines stimulate ovarian cancer cells to secrete MCP1, which promotes macrophage infiltration. Once in the tumor microenvironment, TAMs mature, secrete pro-tumoral cytokines, and facilitate increased tumor growth. Future studies should examine the exact role of TAMs, how sustained adrenergic signaling affects their activity, and how they alter the tumor microenvironment. Our findings implicate MCP1 as another potential therapeutic target, which would inhibit tumor-promoting effects caused by increased adrenergic signaling.

\section{MATERIALS AND METHODS}

\section{Reagents}

Norepinephrine, epinephrine, isoproterenol and dibutyryl-cAMP, propranolol were purchased from SigmaAldrich (St. Louis, MO). Forskolin was purchased from Calbiochem (San Diego, CA). Atenelol, ICI115,881 and SR59230A were purchased from Tocris Biosciences (Minneapolis, MN).

\section{Cell lines and culture conditions}

The derivation and source of the established ovarian cancer cell lines 2774 and SKOV3ip1 have been reported previously [1]. Cells were maintained in RPMI 1640 supplemented with $10 \%$ fetal bovine serum and $0.1 \%$ gentamicin sulfate (Gemini Bioproducts; Calabasas, CA). All cell lines were routinely screened for Mycoplasma species (Fisher; Itasca, IL). All experiments were performed with cell cultures at $70 \%-80 \%$ confluence.

\section{Enzyme-linked immunosorbent assay}

MCP1 and CSF1 protein levels were determined by ELISA using the Quantikine Immunoassay kit (R\&D Systems, Minneapolis, MN) or by Milliplex MAP analyses Millipore, MA) according to the manufacturer's protocol. Samples were assayed in duplicate, and data represent the mean concentration average over triplicate experiments.

\section{Cytokine assay}

Supernatant from cultured ovarian cancer cells were stored at $-20^{\circ} \mathrm{C}$ for batch analyses to measure cytokines. Supernatants were evaluated for cytokines/chemokines using the Milliplex MAP human cytokine/chemokine panel (Millipore, MA). Cytokine levels were measured in $50 \mu \mathrm{L}$ of supernatant by Multiplex cytometric bead array (Multiplex) assay on a Luminex 100 Analyzer (Luminex Corp., Austin, Texas). The inter-variability for all inflammatory cytokines tested was less than $10 \%$, indicating the highly reliability of the Multiplex-Luminex method of cytokine assay.

\section{Quantitative PCR}

For mRNA quantification, ovarian cancer cell or tumor sample total RNA was extracted using RNeasy kit (Qiagen, Valencia, CA). Analysis of mRNA levels was performed on a 7500 Fast Real Time PCR system (Applied Biosystems) with SYBR Green-based real time PCR. Specific Primers for human MCP1 (forward)5'-AGGTGACTGGGGCATTGAT-3', (reverse)-5'GCCTCCAGCATGAAAGTCTC-3' were used. 18S was used as a housekeeping gene.

\section{siRNA preparation and treatment}

Human MCP1-specific siRNA and Control siRNA was purchased from Sigma Genosys (The Woodlands, TX). For in vitro studies, cells were transfected with siRNA that was incorporated into Hiperfect transfection reagent (Qiagen, San Diego, CA) following the manufacturer's protocol. After $48 \mathrm{~h}$, the experiments were carried out. For in vivo studies, siRNA was incorporated into the neutral liposome 1,2-dioleoyl-snglycero-3-phosphatidylcholine (DOPC) as previously described [20]. For each treatment, $3.5 \mu \mathrm{g}$ of siRNA was reconstituted in $200 \mu \mathrm{L}$ of phosphate buffered saline and administered by intravenous injection. The dosing schedule is described in the in vivo tumor model below.

\section{Chronic stress murine model}

We obtained 8- to 12-week-old female athymic nude mice from the National Cancer Institute. All experiments were approved by the Institutional Animal Care and Use Committee at M. D. Anderson Cancer Center. To experimentally induce stress, we used a daily physicalrestraint-stress model as previously described [1, 14]. Tumor cells were injected intraperitoneally into mice in all groups 7 days after the stress procedure began. A total of 10 mice per group were used. Starting 4 days after tumor cell injection, mice were treated with siRNA (control or MCP1-specific, $3.5 \mu \mathrm{g}$ in DOPC every 3 days, intraperitoneally) for the duration of the experiments. Animals were sacrificed 28 days after tumor cell injection. 


\section{Patient demographics}

Following approval by the local institutional review boards, clinical data from primary epithelial ovarian cancer patients from four U.S. academic medical centers were analyzed for this study. Out of 537 initial patients, 462 patients had complete blood count from the time of diagnosis. Of these, 76 patients had available tissue samples from their initial debulking. Primary tumor samples from these patients were analyzed for CD68 expression. Patient and tumor characteristics are included in Supplementary Table 2.

\section{Immunohistochemistry}

Formalin-fixed, paraffin-embedded human samples were stained for CD68. Slides were heated overnight at $65^{\circ} \mathrm{C}$ and then deparaffinized using graded xylenes. Antigen retrieval was performed using Citrate Buffer solution in a vegetable steamer for $40 \mathrm{~min}$ and followed by a 30-min cool-down. Endogenous peroxidase activity was blocked by incubation in 3\% hydrogen peroxide (in methanol) for $12 \mathrm{~min}$. The protein blocking solution was applied for 30 min using an Fc Receptor Blocker (Innovex Biosciences, Richmond, CA). Primary antibodies were applied at a dilution of 1:200 and incubated overnight in a humidity chamber at $4^{\circ} \mathrm{C}$. Secondary antibodies were visualized using the MACH4+ polymer-linked horseradish peroxidase system (Biocare, Concord, CA). 3,3'-Diaminobenzidine was applied, and staining was monitored visually under a bright field microscope ( 3-4 min). Counterstaining was performed using Gill's Hematoxylin for $10 \mathrm{sec}$, followed by washing with phosphate-buffered saline. Slides were dried and mounted using universal mounting medium and then scored by a board-certified pathologist. Counts were determined by the amount of CD68+ cells per field.

\section{Co-immunofluorescence staining for CD-14 and MCP-1}

Paraffin-embedded samples from SKOV3ip1 tumor bearing mice were deparaffinized, permeabilized in cold methanol, incubated overnight with CD-14 and MCP1 antibody, stained with an anti-rabbit and anti-mouse secondary antibodies, respectively, secondary antibody conjugated to a $\mathrm{Cy} 3$ or $\mathrm{Cy} 5$ fluorochrome (Molecular Probes, Eugene, OR), and visualized with a confocal microscope.

\section{Statistical analysis}

We compared continuous variables using either Student's $t$-test or analysis of variance and compared the categorical variables using the chi-squared test. When appropriate, we used a nonparametric test (MannWhitney test) to compare differences. The best cutoff for disease-specific survival was based on ROC curve analysis.
MCP1 levels, CD68+ cells and monocyte levels were compared using spearman's correlation analysis. Survival curves were analyzed by the Kaplan-Meier method, and statistical significance was determined by the log-rank test. We considered $P<0.05$ to be significant.

\section{ACKNOWLEDGMENTS}

RLS, HJD, and RAP are supported by an NIH T32 Training Grant CA101642. SKL was supported by the following grants: CA140933 and CA104825. This work was supported by a Research Training Award from the Cancer Prevention and Research Institute of Texas (CPRIT RP140106) to AN. GLB was supported by the Ovarian Cancer Research Foundation, DOD-PC0-80359-P1, NIH/ NCI P50 CA093459, 5RC2GM09259, 5U54CA151668 and U54 CA 096300-08. This work was also supported, in part, by NIH grants (CA109298, P50CA083639, P50CA098258, U54CA151668, CA016672, U54CA96300 and U54CA96297), Ovarian Cancer Research Fund Program Project Development Grant, Department of Defense (OC073399, W81XWH-10-1-0158, OC100237), the Bettyann Asche Murray Distinguished Professorship, the RGK Foundation, the Gilder Foundation, and the Blanton-Davis Ovarian Cancer Research Program.

\section{CONFLICT OF INTEREST}

The authors declare no potential conflicts of interest.

\section{REFERENCES}

1. Thaker PH, Han LY, Kamat AA, Arevalo JM, Takahashi R, Lu C, Jennings NB, Armaiz-Pena G, Bankson JA, Ravoori M, Merritt WM, Lin YG, Mangala LS, Kim TJ, Coleman RL, Landen CN, et al. Chronic stress promotes tumor growth and angiogenesis in a mouse model of ovarian carcinoma. Nat Med. 2006; 12:939-944.

2. Antoni MH, Lutgendorf SK, Cole SW, Dhabhar FS, Sephton SE, McDonald PG, Stefanek M, Sood AK. The influence of bio-behavioural factors on tumour biology: pathways and mechanisms. Nat Rev Cancer. 2006; 6:240-248.

3. Armaiz-Pena GN, Lutgendorf SK, Cole SW, Sood AK. Neuroendocrine modulation of cancer progression. Brain Behav Immun. 2009; 23:10-15.

4. Sood AK, Armaiz-Pena GN, Halder J, Nick AM, Stone RL, $\mathrm{Hu}$ W, Carroll AR, Spannuth WA, Deavers MT, Allen JK, Han LY, Kamat AA, Shahzad MM, McIntyre BW, DiazMontero CM, Jennings NB, et al. Adrenergic modulation of focal adhesion kinase protects human ovarian cancer cells from anoikis. The Journal of clinical investigation. 2010; 120:1515-1523.

5. Sood AK, Bhatty R, Kamat AA, Landen CN, Han L, Thaker PH, Li Y, Gershenson DM, Lutgendorf S, Cole SW. Stress hormone-mediated invasion of ovarian cancer cells. 
Clinical cancer research: an official journal of the American Association for Cancer Research. 2006; 12:369-375.

6. Shahzad MM, Arevalo JM, Armaiz-Pena GN, Lu C, Stone RL, Moreno-Smith M, Nishimura M, Lee JW, Jennings NB, Bottsford-Miller J, Vivas-Mejia P, Lutgendorf SK, Lopez-Berestein G, Bar-Eli M, Cole SW, Sood AK. Stress effects on FosB- and interleukin-8 (IL8)driven ovarian cancer growth and metastasis. The Journal of biological chemistry. 2010; 285:35462-35470.

7. Landen $\mathrm{CN}$ Jr., Lin YG, Armaiz Pena GN, Das PD, Arevalo JM, Kamat AA, Han LY, Jennings NB, Spannuth WA, Thaker PH, Lutgendorf SK, Savary CA, Sanguino AM, Lopez-Berestein G, Cole SW, Sood AK. Neuroendocrine modulation of signal transducer and activator of transcription-3 in ovarian cancer. Cancer Res. 2007; 67:10389-10396.

8. Nilsson MB, Armaiz-Pena G, Takahashi R, Lin YG, Trevino J, Li Y, Jennings N, Arevalo J, Lutgendorf SK, Gallick GE, Sanguino AM, Lopez-Berestein G, Cole SW, Sood AK. Stress hormones regulate interleukin-6 expression by human ovarian carcinoma cells through a Src-dependent mechanism. J Biol Chem. 2007; 282:29919-29926.

9. Solinas G, Germano G, Mantovani A, Allavena P. Tumorassociated macrophages (TAM) as major players of the cancer-related inflammation. J Leukoc Biol. 2009; 86:1065-1073.

10. Turpin J.A., Lopez-Berestein G. Differentiation, Maturation and Activation of monocytes and Macrophages: Functional activity is defined by a continuum of maturation. PP. 71-100.

11. Pollard JW. Tumour-educated macrophages promote tumour progression and metastasis. Nat Rev Cancer. 2004; 4:71-78.

12. Lutgendorf SK, Lamkin DM, Jennings NB, Arevalo JM, Penedo F, DeGeest K, Langley RR, Lucci JA 3rd, Cole SW, Lubaroff DM, Sood AK. Biobehavioral influences on matrix metalloproteinase expression in ovarian carcinoma. Clin Cancer Res. 2008; 14:6839-6846.

13. Sloan EK, Priceman SJ, Cox BF, Yu S, Pimentel MA, Tangkanangnukul V, Arevalo JM, Morizono K, Karanikolas BD, Wu L, Sood AK, Cole SW. The sympathetic nervous system induces a metastatic switch in primary breast cancer. Cancer Res. 2010; 70:7042-7052.

14. Armaiz-Pena GN, Allen JK, Cruz A, Stone RL, Nick AM, Lin YG, Han LY, Mangala LS, Villares GJ, Vivas-Mejia P, Rodriguez-Aguayo C, Nagaraja AS, Gharpure KM, Wu Z, English RD, Soman KV, et al. Src activation by betaadrenoreceptors is a key switch for tumour metastasis. Nature communications. 2013; 4:1403.

15. Gyorffy B, Lanczky A, Szallasi Z. Implementing an online tool for genome-wide validation of survival-associated biomarkers in ovarian-cancer using microarray data from 1287 patients. Endocrine-related cancer. 2012; 19:197-208.

16. Conti I, Rollins BJ. CCL2 (monocyte chemoattractant protein-1) and cancer. Semin Cancer Biol. 2004; 14:149-154.

17. Becker LC. Yin and yang of MCP-1. Circulation research. 2005; 96:812-814.

18. Negus RP, Stamp GW, Relf MG, Burke F, Malik ST, Bernasconi S, Allavena P, Sozzani S, Mantovani A, Balkwill FR. The detection and localization of monocyte chemoattractant protein-1 (MCP-1) in human ovarian cancer. The Journal of clinical investigation. 1995; 95:2391-2396.

19. Lutgendorf SK, DeGeest K, Sung CY, Arevalo JM, Penedo F, Lucci J 3rd, Goodheart M, Lubaroff D, Farley DM, Sood AK, Cole SW. Depression, social support, and beta-adrenergic transcription control in human ovarian cancer. Brain Behav Immun. 2009; 23:176-183.

20. Landen CN Jr, Chavez-Reyes A, Bucana C, Schmandt R, Deavers MT, Lopez-Berestein G, Sood AK. Therapeutic EphA2 gene targeting in vivo using neutral liposomal small interfering RNA delivery. Cancer Res. 2005; 65:6910-6918. 